\title{
20.5 kA Current Leads for ATLAS Barrel Toroid Superconducting Magnets
}

\author{
A. V. Dudarev, H. H. J. ten Kate, E. W. Boxman, V. E. Keilin, N. Ph. Kopeikin, I. A. Kovalev, A. N. Kuljamzin, \\ V. R. Romanovskii, V. I. Shcherbakov, I. O. Shugaev, and V. V. Stepanov
}

\begin{abstract}
Three pairs of $20.5 \mathrm{kA}$ current leads for the ATLAS Toroid Magnets have been designed, manufactured and tested at Kurchatov Institute. The current leads have a high mechanical reliability and the vacuum tightness under 30 bars of internal pressure. The insulation between the current carrying parts and the mounting flange, the hydraulic connections and the temperature gauges withstand the overvoltage of at least $2 \mathrm{kV}$. The current leads are fully equipped with diagnostics needed for safety and control. The current leads were tested up to $24 \mathrm{kA}$. According to CERN's specification they were also tested in the absence of any cooling at very slow current discharge rate $(5 \mathrm{~A} / \mathrm{s})$ from $20.5 \mathrm{kA}$ to zero without any excessive overheating. Nowadays the current leads are successfully used at the ATLAS Magnet Test Facility at CERN.
\end{abstract}

Index Terms-ATLAS, cryogenic test, current leads, design.

\section{INTRODUCTION}

$\mathbf{T}$ HREE pairs of current leads for the superconducting Barrel Toroid (and the relevant model windings [1]) of the detector ATLAS being constructed at CERN were designed, manufactured and tested at Kurchatov Institute, Moscow, Russia. The operating current is $20.5 \mathrm{kA}$ in a practically continuous mode, the maximum current is $24 \mathrm{kA}$. The main requirement was a maximum reliability at a reasonably low heat leak (but not necessarily close to a theoretical minimum). The current leads must withstand 30 bar internal pressure without losing a practically absolute vacuum tightness. Any use of soft-solders was not allowed. All vacuum-tight seals should be done only by electron-beam or tungsten inert gas welding. In the case of a sudden interruption of a gaseous helium coolant flow, the current leads must keep the current for a rather long time without an unacceptable overheating. This requirement was quantitatively formulated as an ability to withstand without any coolant flow a very low $\left(5 \mathrm{~A} \cdot \mathrm{s}^{-1}\right)$ current decay from the operating value $(20.5 \mathrm{kA})$ to zero. The maximum heat leak at $20.5 \mathrm{kA}$ must not exceed $30 \mathrm{~W}$ (or to correspond to the coolant flow $1.5 \mathrm{~g} / \mathrm{s}$ per lead, i.e., to approximately $50 \%$ higher value than the theoretical minimum [2]). The pressure drop must not exceed 50 mbar. The lead-to-ground breakage voltage must be higher than $2 \mathrm{kV}$. The height of the heat exchanger part was limited to approximately $1300 \mathrm{~mm}$.

Manuscript received September 24, 2001.

A. V. Dudarev, H. H. J. ten Kate, and E. W. Boxman are with CERN, Geneva 23, CH-1211, Switzerland (e-mail: Alexey.Dudarev@ cern.ch).

V. E. Keilin, N. Ph. Kopeikin, I. A. Kovalev, A. N. Kuljamzin, V. R. Romanovskii, V. I. Shcherbakov, I. O. Shugaev, and V. V. Stepanov are with the Kurchatov Institute, Moscow, 123182, Russia (e-mail: kev@isssph.kiae.ru).

Publisher Item Identifier S 1051-8223(02)04181-7.

\section{CURRENT LEADS DESIGN}

\section{A. Choice of Material}

Several options for metals to be used in the heat exchanger part of the current leads were considered. Pure copper that is the most widely used metal for current leads was rejected for the two following (inherently interconnected) reasons: i) its too low resistivity at low temperatures results in a too small lead cross-section and, hence, in its mass at a reasonably long length, especially taking into account the required ability to withstand the coolant loss for a long time periods; ii) the sharp resistivity versus temperature dependence of a pure copper prevents its use in current leads subjected to an overloading (the ability to carry high current in the absence of the coolant flow represents actually an overloading mode of operation).

It was shown long ago [3] that the efficiency of current leads at operating conditions does not depend on what metal they are made of, provided the cooling efficiency is the same and the length-to-cross-section ratio is properly chosen. However, as it was shown in [4], if current leads must operate in different, especially highly overloaded, conditions, metal alloys (like brass or even stainless steel) with their weak resistivity versus temperature dependence are preferable. However, in this case the relatively high resistivity of brass (to say nothing of stainless steel) would result in a too large current lead cross-section at a given length $(1300 \mathrm{~mm})$. The calculations have shown that a reasonable compromise would be a metal with a copper-like resistivity at $300 \mathrm{~K}$ (i.e., about $20 \mathrm{n} \Omega \cdot \mathrm{m}$ ) and RRR about 6 . These requirements can be met with the $\mathrm{Cu}-0.3 \mathrm{wt}$.\% Ni alloy. $2 \mathrm{~mm}$ diameter wires of this alloy were specially manufactured by Bochvar Institute for the ATLAS current leads.

\section{B. Design}

The current lead design is schematically shown in Fig. 1. Its heat exchanger part consists of $10602 \mathrm{~mm}$ diameter $1300 \mathrm{~mm}$ length wires made of the above mentioned $\mathrm{Cu}-0.3$ wt. $\% \mathrm{Ni}$ alloy.

The wires surround a central stainless steel tube. The both ends of the wires are inserted (and then brazed) in holes drilled in upper and lower copper rings machined in the massive warm and cold copper terminals. The photo of the heat exchanger in the process of current lead assembly is shown in Fig. 2. The wires are brazed in holes in the copper rings with a copper-based hard solder (melting point $640^{\circ} \mathrm{C}$ ). The set of the wires is tightly embedded into a $150 \mathrm{~mm}$ diameter $2.5 \mathrm{~mm}$ wall thickness stainless steel tube. All vacuum-tight connections between copper and stainless steel parts are done with electron-beam welding. A G-10 flange is used for the current lead-to-ground electrical insulation. The current lead design provides for minimizing the 


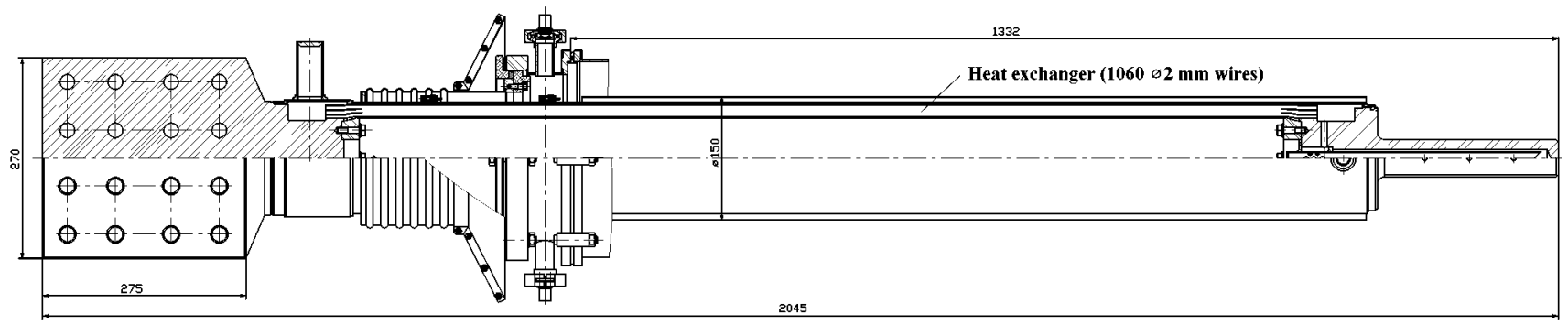

Fig. 1. The current lead design.

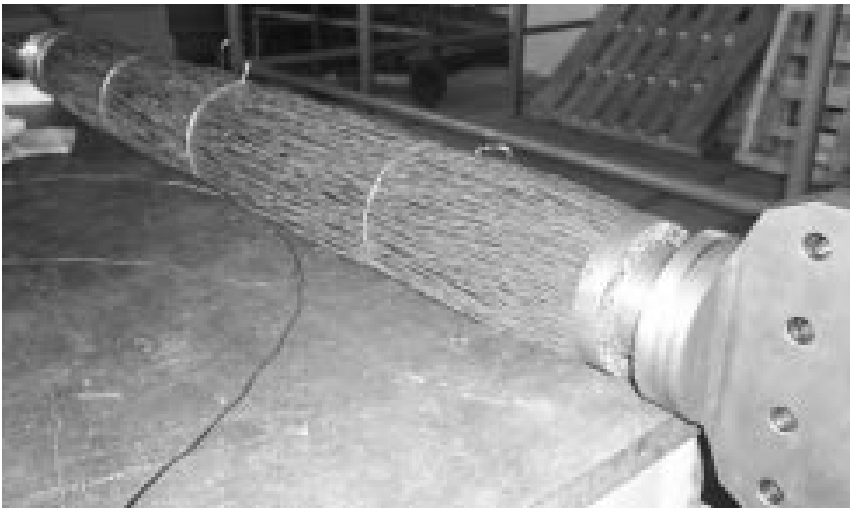

Fig. 2. The heat exchanger.

possibility of a frost and water accumulation at the warm terminals. The current leads are equipped with potential taps and thermometers. The latter ones are placed in the "burn through" region, in the middle of the length, and at the cold end. In addition, differential pressure gauges as well as heaters at the warm terminals are placed.

\section{Calculations}

For the described heat exchanger geometry, the calculations of heat leaks, voltages and temperature distribution were performed. The results, presented in Figs. 3 and 4, were obtained by solving a set of equations, written for one lead:

$$
\begin{aligned}
\frac{d}{d x}\left(k \frac{d T}{d x}\right)-\frac{h p}{S}\left(T-T_{g}\right)+\frac{I^{2}}{S^{2}} \rho(T) & =0, \\
C_{p} m \frac{d T_{g}}{d x} & =h p\left(T-T_{g}\right) .
\end{aligned}
$$

Here $T$ is the temperature, $x$ is the spatial coordinate along the lead, $k(T)$ is the leads thermal conductivity, $h$ is the temperature dependent heat transfer coefficient to GHe, $p$ is the cooled perimeter, $S$ is the lead cross-section, $\rho(T)$ is the electric resistivity, $C_{p}$ is the specific heat of helium gas, $m$ is the mass flow rate of GHe. Subscript $g$ stands for helium gas. The equations (1) describe the energy balance of an element of the lead. The boundary conditions at the cold $(x=0)$ and warm $(x=l)$ terminals are:

$$
T(0)=T_{0}, T_{\mathrm{g}}(0)=T_{0}, T(l)=T_{l} .
$$

For the lead under consideration the minimum heat transfer coefficient was conservatively determined by the minimum value of the Nusselt number:

$$
\mathrm{Nu}=\frac{h d}{k_{g}(T)}=3.66,
$$

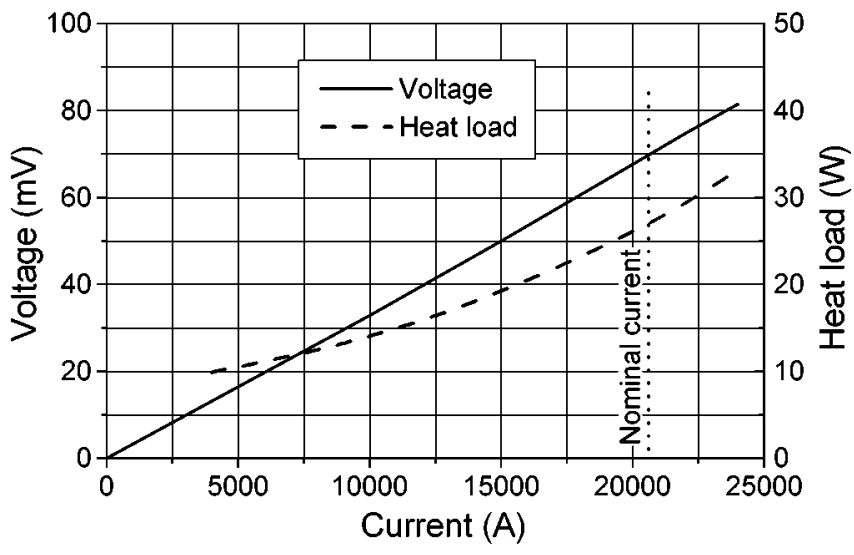

Fig. 3. Voltage and heat load calculation results (per one lead).

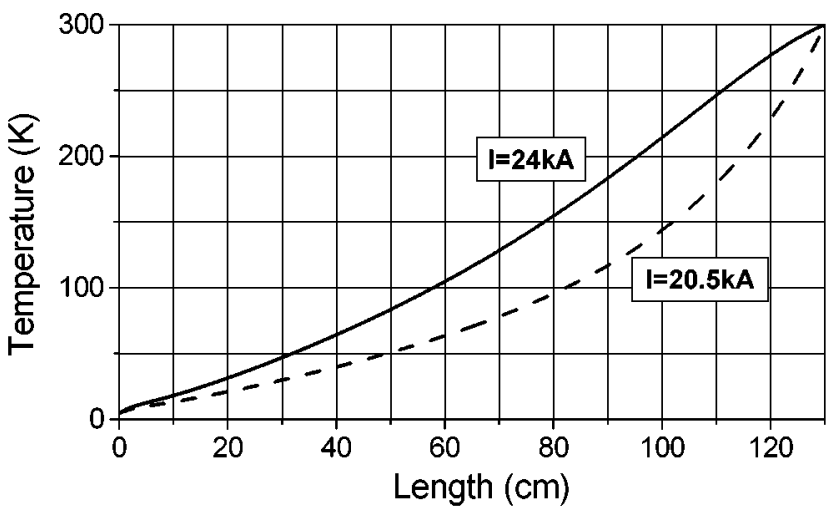

Fig. 4. Calculated temperature distribution.

where $d=4 \Delta S / F$ is the hydraulic diameter corresponding to the free cross-section of the annulus $\Delta S$ and the wetted perimeter $F$ which is equal to the sum of the perimeters of all wires and tubes. The helium and wire material properties are temperature dependent. The properties were taken as:

$$
\begin{aligned}
k_{g}(T)= & 2.4115 \times 10^{-3} T_{g}^{0.7305}\left[\frac{\mathrm{W}}{\mathrm{m} \cdot \mathrm{K}}\right], \\
C_{p}= & 5.2 \times 10^{3}\left[\frac{\mathrm{J}}{\mathrm{kg} \cdot \mathrm{K}}\right], \\
\rho(T)= & 9.26 \times 10^{-14} T^{2}+2.64 \times 10^{-11} T \\
& +3.75 \times 10^{-9}[\Omega \cdot \mathrm{m}] .
\end{aligned}
$$




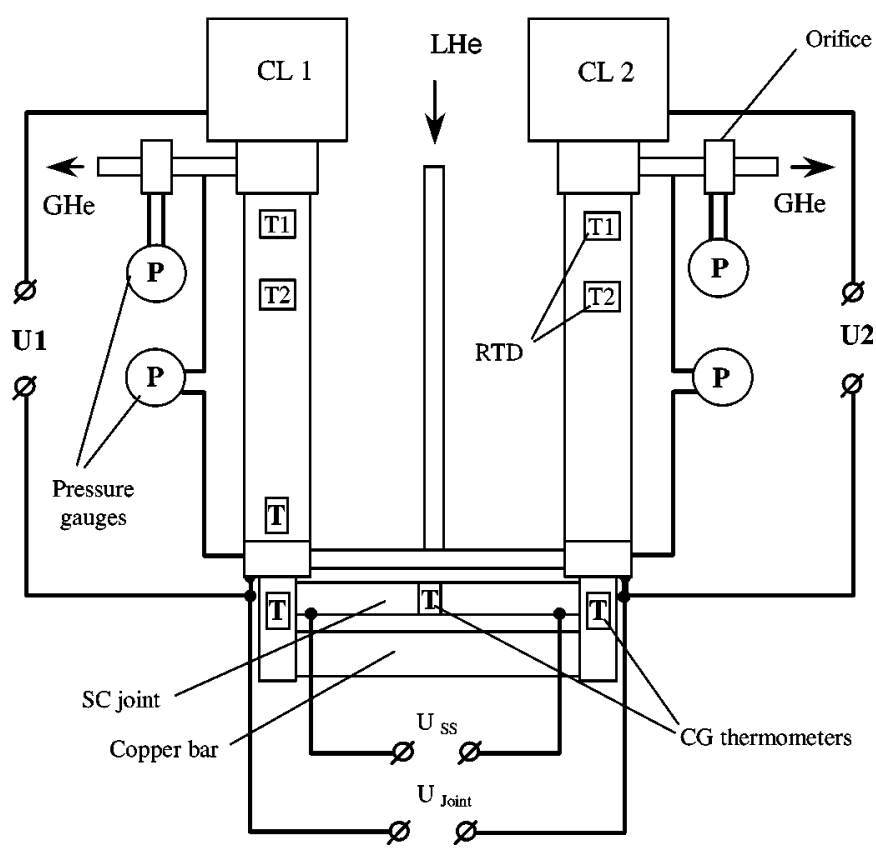

Fig. 5. Experimental setup.

The thermal conductivity of copper was taken from the Wiedemann-Franz-Lorentz law [2]:

$$
k(T)=2.45 \times 10^{-8} \frac{T}{\rho(T)}\left[\frac{\mathrm{W}}{\mathrm{m} \cdot \mathrm{K}}\right] .
$$

The minimum mass flow rate $m$ is connected with the heat leak $Q$ to the LHe region through the following relationship, valid for the self-sustained condition at the cold terminal:

$$
Q=\left.k(T) \cdot S \cdot \frac{d T}{d x}\right|_{x=0}=m r .
$$

Here $r$ is the LHe latent heat of vaporization. Calculations were carried out for the following parameters of the lead: $T_{0}=4.5 \mathrm{~K}$, $T_{l}=300 \mathrm{~K}, l=1.3 \mathrm{~m}$.

\section{Test PRoCEDURES AND RESUlts}

During and after manufacturing, the current leads and their parts underwent a careful inspection and different quality assurance tests. All welded vacuum-tight seals were subjected to several thermocycles from room to liquid $\mathrm{N}_{2}$ temperature, pressurizing up to 30 bars and subsequent helium leak detector checking. All electric insulators underwent dc electrical strength tests up to $2 \mathrm{kV}$. In all such tests, the leakage current was less than $2 \mu \mathrm{A}$, corresponding to the insulation resistance higher than $1 \mathrm{G} \Omega$ per current lead. At the final cryogenic stage of testing, the helium mass flow per lead was measured with a standard orifice. The voltage drop along each current lead, the temperatures in three points along the lead length as well as the pressure drop were measured. The scheme of the final tests at Kurchatov Institute is given in Fig. 5. Each pair of current leads was placed in a high vacuum vessel. The vacuum-tightness of the current leads was checked by a high-sensitivity helium leak detector.

The cold terminals were electrically short-circuited with massive copper bars and NbTi based monolithic conductors. Liquid

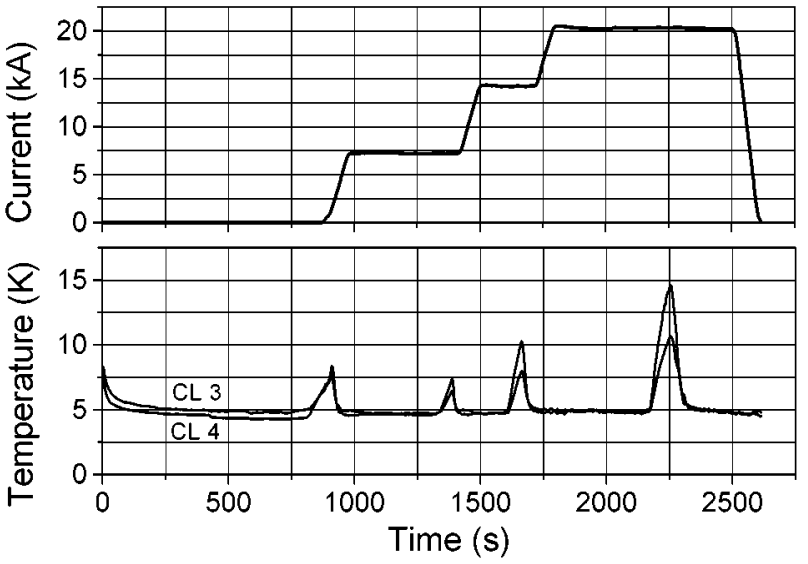

Fig. 6. Current and cold terminal temperature histories.


Fig. 7. Current and temperatures near the warm terminal in the absence of cooling.

helium entered the cold terminals from a storage vessel. At several current values $(0.7 \mathrm{kA}, 14 \mathrm{kA}, 20.5 \mathrm{kA})$ a minimum mass flow was fixed corresponding to a start of cold terminal temperature increase from 4.5 to $5 \mathrm{~K}$. In the beginning of this process, the helium flow was used, which was somewhat larger than the optimal one. As a result, part of the heat exchanger was filled with LHe. Then the LHe supply from the storage vessel was closed and LHe evaporated out of the heat exchanger. The end of evaporation was fixed by an abrupt increase of the cold terminal temperature. The preceding evaporation rate was considered as a mass flow that corresponds to the given current. The method is very time efficient, but results (as the measurements in CERN have shown) in somewhat (10 to 15\%) lower values of mass flow rates. It can be explained by the fact that in the described procedure the heat exchanger/temperature distribution had not enough time to become thermally equilibrium, and the temperatures were somewhat lower than the equilibrium ones. For the same reason the voltage drops measured at Kurchatov Institute were lower than those measured at CERN. After mass flow rates measurements were over, the current leads were charged up to $24 \mathrm{kA}$ for 10 minutes. In Fig. 6 as an example, the current and cold terminals temperature histories are shown for the 2nd pair of current leads (CL3 and CL4). The test results in case of coolant absence are shown in Fig. 7, when the initial cold end temperature was approximately $5 \mathrm{~K}$. It is seen that at 


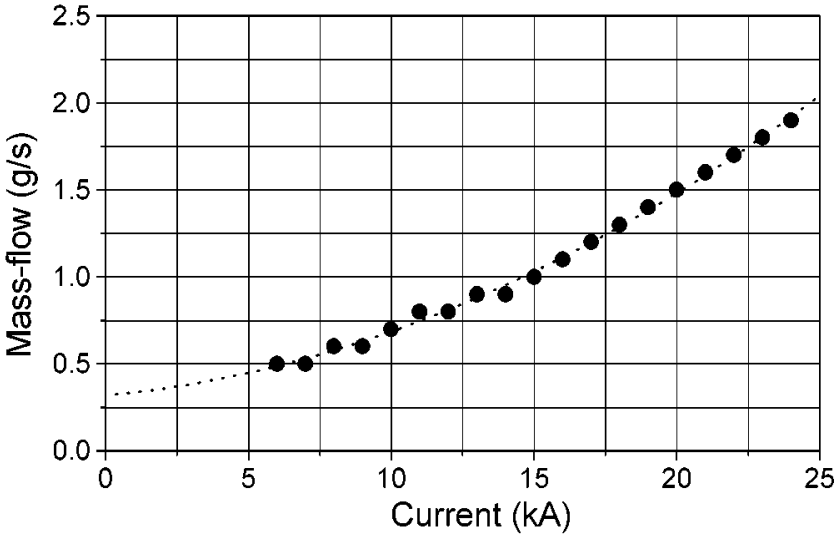

Fig. 8. Mass-flow rate (per one lead) versus current.

the end of current decrease there is no overheating. It must be also noted that the overheating tests were also carried out for the case, when the cold terminal was cooled only to liquid $\mathrm{N}_{2}$ initial temperature and even in the case of the whole current lead being initially at room temperature. In each of these cases the unacceptable overheating was not observed (maximum temperature did not exceed $150^{\circ} \mathrm{C}$ ). The measured pressure drop along a current lead at approximately $1.5 \mathrm{~g} / \mathrm{s}$ was about $10 \mathrm{mbar}$ (i.e., much less than acceptable 50 mbar). At the end of each cryogenic test, the electric strength of the lead-to-ground insulation was measured. In all cases it was better than $2 \mathrm{kV}$. The cryogenic tests carried out at Kurchatov Institute have shown that all characteristics of the three pairs of current leads are the same within at least $10 \%$. The first pair of current leads was tested at CERN together with B00 model windings [5], [6]. The necessary mass flow was found from the condition of the time-stable voltage drop along the current lead. Mass flow equal to $1.5 \mathrm{~g} / \mathrm{s}$ corresponds to the time-stable voltage drop equal to approximately $70 \mathrm{mV}$ per lead. In Fig. 8 the mass flow rate versus current dependence obtained in CERN's tests is shown. The unusually large ratio of the mass flow rates at high and low (or zero) currents is explained by two reasons: i) imperfection of heat transfer at high currents and ii) using low RRR copper (instead of pure one) in the current lead heat exchanger.

\section{CONCLUSION}

Three pairs of current leads for the superconducting magnets (and the relevent model windings) of the ATLAS detector are designed, manufactured and tested. The current leads fully meet the specification. Moreover, as the test results show, the current leads are somewhat "overdesigned," and are capable to carry currents larger than $24 \mathrm{kA}$. The electrical, thermal and hydraulic properties of all 6 leads are practically identical. Nowadays the current leads are being successfully used at the CERN's test facility to test model windings for the ATLAS magnet program. A visual confirmation of the current leads efficiency is the fact, that neither frost nor water is accumulated at their warm terminals at $20.5 \mathrm{kA}$ and $1.5 \mathrm{~g} / \mathrm{s}$, and there is no necessity to warm up the terminals with switching on the corresponding heaters.

\section{ACKNOWLEDGMENT}

The authors would like to thank the superconducting team of Bochvar Institute for the production and supply of copper alloy wires. They also appreciate Prof. N. A. Chernoplekov for his interest and support of this work. Many thanks to I. O. Shchegolev for his assistance in the preparation of the paper.

\section{REFERENCES}

[1] H. H. J. ten Kate and ATLAS Collaboration, "The superconducting magnet system for the ATLAS detector at CERN," IEEE Trans. Appl. Superconduct., vol. 10, pp. 347-352, Mar. 2000.

[2] M. N. Wilson, Superconducting Magnets. Oxford: Oxford Univ. Press, 1983.

[3] V. E. Keilin and E. Yu. Klimenko, "Investigation into high current leads in liquid helium applications," Cryogenics, vol. 6, pp. 222-228, 1966.

[4] V. E. Keilin and A. V. Gavrilin, "Overloaded current leads," in Proc. 15th Intern. Conf. Magnet Technology (MT-15), L. Liangzhen, S. Guoliao, and Y. Luguang, Eds. Beijing: Science Press, 1998, pp. 81-83.

[5] O. P. Anashkin, V. E. Keilin, and V. V. Lysenko, "The B00 model coil in the ATLAS magnet test facility," IEEE Trans. Appl. Superconduct., vol. 11, pp. 1582-1585, Mar. 2001.

[6] E. M. Boxman, A. V. Dudarev, A. Janssen, and H. H. J. ten Kate, "The normal zone propagation in ATLAS B00 model coil," IEEE Trans. Appl. Superconduct., submitted for publication. 